Eric Vittinghoff $\bullet$ David V. Glidden

Stephen C. Shiboski $\bullet$ Charles E. McCulloch

\title{
Regression Methods in Biostatistics
}

Linear, Logistic, Survival, and Repeated Measures Models

Second edition

盟 Springer 


\section{Contents}

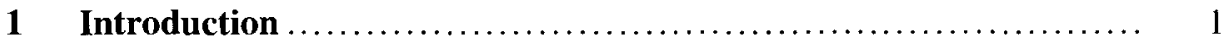

1.1 Example: Treatment of Back Pain ........................ 1

1.2 The Family of Multipredictor Regression Methods ............ 2

1.3 Motivation for Multipredictor Regression .................. 3

1.3.1 Prediction...................................... 3

1.3.2 Isolating the Effect of a Single Predictor ............. 3

1.3.3 Understanding Multiple Predictors ................ 4

$1.4 \quad$ Guide to the Book................................... 4

2 Exploratory and Descriptive Methods $\ldots \ldots \ldots \ldots \ldots \ldots \ldots \ldots \ldots \ldots$

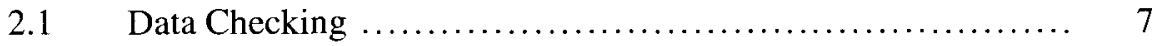

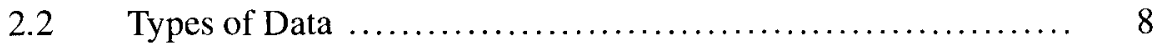

$2.3 \quad$ One-Variable Descriptions............................... 9

2.3.1 Numerical Variables ........................... 9

2.3.2 Categorical Variables ......................... 16

2.4 Two-Variable Descriptions ........................... 17

2.4.1 Outcome Versus Predictor Variables ............... 17

2.4.2 Continuous Outcome Variable ..................... 18

2.4.3 Categorical Outcome Variable ................... 21

$2.5 \quad$ Multivariable Descriptions ......................... 22

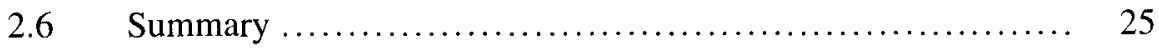

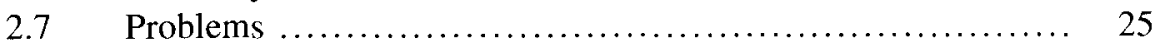

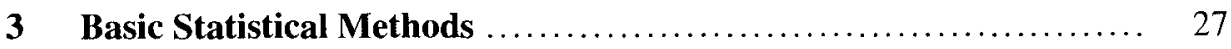

$3.1 t$-Test and Analysis of Variance ........................ 27

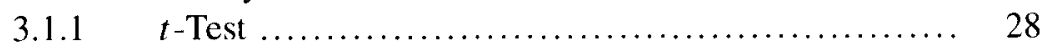

3.1.2 One- and Two-Sided Hypothesis Tests .............. 28

3.1.3 Paired $t$-Test.................................. 29

3.1.4 One-Way Analysis of Variance.................... 30

3.1.5 Pairwise Comparisons in ANOVA .................. 30

3.1.6 Multi-way ANOVA and ANCOVA ................ 31

3.1.7 Robustness to Violations of Normality Assumption ... 31 
3.1.8 Nonparametric Alternatives ................... 32

3.1.9 Equal Variance Assumption .................... 32

3.2 Correlation Coefficient ............................... 33

3.2.1 Spearman Rank Correlation Coefficient ............. 34

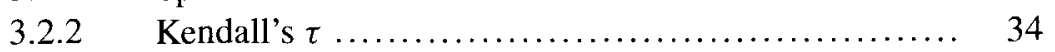

3.3 Simple Linear Regression Model ......................... 35

3.3.1 Systematic Part of the Model................... 35

3.3.2 Random Part of the Model ........................ 36

3.3.3 Assumptions About the Predictor ................. 37

3.3.4 Ordinary Least Squares Estimation ............... 38

3.3.5 Fitted Values and Residuals ..................... 39

$3.3 .6 \quad$ Sums of Squares ............................ 39

3.3.7 Standard Errors of the Regression Coefficients ...... 40

3.3.8 Hypothesis Tests and Confidence Intervals .......... 40

3.3.9 Slope, Correlation Coefficient, and $R^{2} \ldots \ldots \ldots \ldots \ldots, 42$

3.4 Contingency Table Methods for Binary Outcomes............ 42

3.4.1 Measures of Risk and Association for

Binary Outcomes .......................... 43

3.4.2 Tests of Association in Contingency Tables ......... 46

3.4.3 Predictors with Multiple Categories .............. 48

$\begin{array}{ll}3.4 .4 & \text { Analyses Involving Multiple Categorical } \\ & \text { Predictors } \ldots \ldots \ldots \ldots \ldots \ldots \ldots \ldots \ldots \ldots \ldots \ldots \ldots \ldots \ldots \ldots\end{array}$

3.4.5 Collapsibility of Standard Measures of Association ... 52

3.5 Basic Methods for Survival Analysis ..................... 54

3.5.1 Right Censoring.............................. 54

3.5.2 Kaplan-Meier Estimator of the Survival Function ..... 55

3.5.3 Interpretation of Kaplan-Meier Curves ............. 57

3.5.4 Median Survival ............................ 58

3.5.5 Cumulative Event Function ...................... 59

3.5.6 Comparing Groups Using the Logrank Test .......... 60

3.6 Bootstrap Confidence Intervals........................ 62

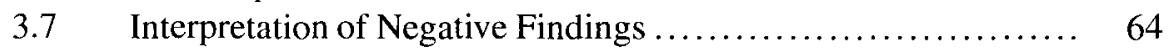

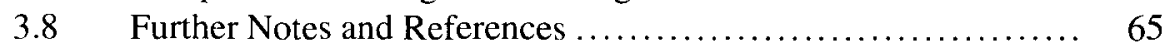

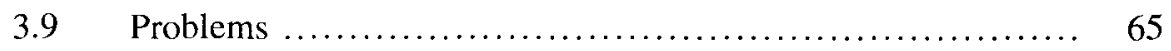

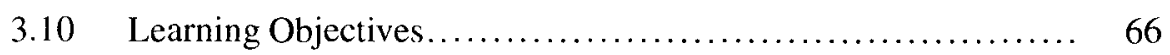

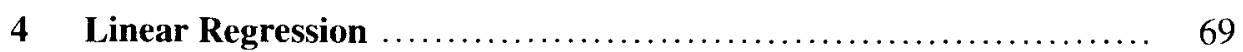

4.1 Example: Exercise and Glucose ......................... 70

4.2 Multiple Linear Regression Model....................... 72

4.2.1 Systematic Part of the Model ...................... 72

4.2.2 Random Part of the Model ...................... 73

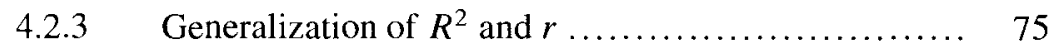

4.2.4 Standardized Regression Coefficients ............. 75

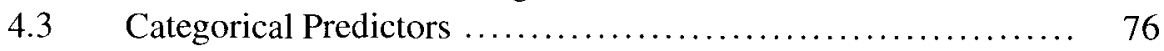

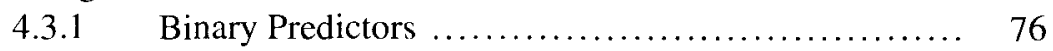


4.3.2 Multilevel Categorical Predictors ............... 77

4.3.3 The $F$-Test .............................. 81

4.3.4 Multiple Pairwise Comparisons Between Categories .. 81

4.3.5 Testing for Trend Across Categories ............... 84

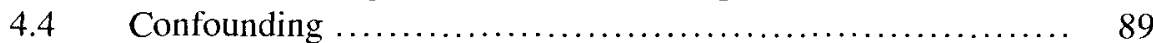

4.4.1 Range of Confounding Patterns .................. 90

4.4.2 Confounding Is Difficult to Rule Out ............... 91

4.4.3 Adjusted Versus Unadjusted $\hat{\beta}$ s ................. 92

4.4.4 Example: BMI and LDL....................... 93

$4.5 \quad$ Mediation.............................................. 94

4.5.1 Indirect Effects via the Mediator .................. 95

4.5.2 Overall and Direct Effects ........................ 95

4.5.3 Percent Explained ............................ 96

4.5.4 Example: BMI, Exercise, and Glucose ............ 96

4.5.5 Pitfalls in Evaluating Mediation................... 97

$4.6 \quad$ Interaction ................................................. 99

4.6.1 Example: Hormone Therapy and Statin Use ......... 100

4.6.2 Example: BMI and Statin Use.................... 102

4.6.3 Interaction and Scale.......................... 105

4.6.4 Example: Hormone Therapy and Baseline LDL ...... 106

4.6.5 Details ....................................... 107

4.7 Checking Model Assumptions and Fit ................... 108

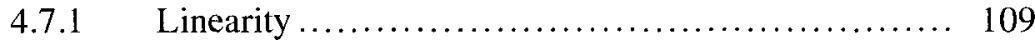

4.7.2 Normality $\ldots \ldots \ldots \ldots \ldots \ldots \ldots \ldots \ldots \ldots \ldots \ldots \ldots, 116$

4.7.3 Constant Variance ........................... 119

4.7.4 Outlying, High Leverage, and Influential Points ...... 124

4.7.5 Interpretation of Results for $\mathrm{Log}$

Transformed Variables........................... 128

4.7.6 When to Use Transformations.................... 129

4.8 Sample Size, Power, and Detectable Effects................ 130

4.8.1 Calculations Using Standard Errors Based

on Published Data............................ 133

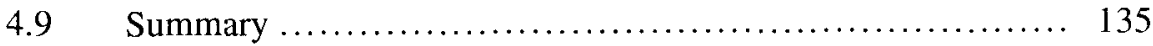

4.10 Further Notes and References ........................ 135

4.10.1 Generalized Additive Models ................... 136

4.11 Problems ........................................ 136

4.12 Learning Objectives............................. 138

$5 \quad$ Logistic Regression ......................................... 139

$5.1 \quad$ Single Predictor Models ................................ 140

5.1.1 Interpretation of Regression Coefficients ............ 144

5.1.2 Categorical Predictors .......................... 146

$5.2 \quad$ Multipredictor Models ............................. 150

5.2.1 Likelihood Ratio Tests ...................... 154

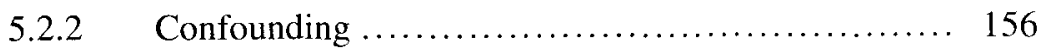


5.2.3 Mediation...................................... 158

$5.2 .4 \quad$ Interaction .................................... 160

$5.2 .5 \quad$ Prediction..................................... 165

5.2.6 Prediction Accuracy ............................. 166

$5.3 \quad$ Case-Control Studies ....................................... 168

5.3.1 Matched Case-Control Studies .................... 171

$5.4 \quad$ Checking Model Assumptions and Fit .................... 173

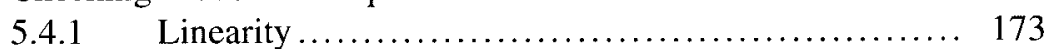

5.4.2 Outlying and Influential Points.................... 175

5.4.3 Model Adequacy .............................. 177

5.4.4 Technical Issues in Logistic Model Fitting ........... 179

5.5 Alternative Strategies for Binary Outcomes ................. 180

5.5.1 Infectious Disease Transmission Models ............ 181

5.5.2 Pooled Logistic Regression ..................... 183

5.5.3 Regression Models Based on Risk

Differences and Relative Risks.................... 186

5.5.4 Exact Logistic Regression ........................ 188

5.5.5 Nonparametric Binary Regression .................. 189

5.5.6 More Than Two Outcome Levels ................... 190

$5.6 \quad$ Likelihood ......................................... 192

5.7 Sample Size, Power, and Detectable Effects................ 194

$5.8 \quad$ Summary ........................................ 199

$5.9 \quad$ Further Notes and References .......................... 200

5.10 Problems ............................................ 200

5.11 Learning Objectives................................. 202

6 Survival Analysis ............................................ 203

6.1 Survival Data ........................................ 203

6.1.1 Why Linear and Logistic Regression Would not Work .................................. 203

6.1.2 Hazard Function ................................ 204

6.1.3 Hazard Ratio .................................. 205

6.1.4 Proportional Hazards Assumption ................. 207

6.2 Cox Proportional Hazards Model .......................... 207

6.2.1 Proportional Hazards Models ..................... 207

6.2.2 Parametric Versus Semi-parametric Models.......... 208

6.2.3 Hazard Ratios, Risk, and Survival Times............ 211

6.2.4 Hypothesis Tests and Confidence Intervals.......... 212

6.2.5 Binary Predictors ............................. 213

6.2.6 Multilevel Categorical Predictors .................. 213

6.2.7 Continuous Predictors ............................ 217

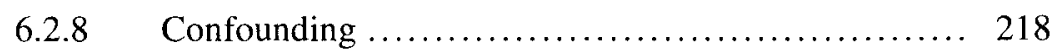

$6.2 .9 \quad$ Mediation..................................... 219

$6.2 .10 \quad$ Interaction ................................... 220

$6.2 .11 \quad$ Model Building .............................. 222 
6.2.12 Adjusted Survival Curves for Comparing Groups...... 222

6.2.13 Predicted Survival for Specific Covariate Patterns .... 224

6.3 Extensions to the Cox Model ............................. 225

6.3.1 Time-Dependent Covariates ................... 225

6.3.2 Stratified Cox Model ........................... 228

$6.4 \quad$ Checking Model Assumptions and Fit ................... 231

6.4.1 Log-Linearity of the Hazard Function ............. 231

6.4.2 Proportional Hazards ......................... 232

6.5 Competing Risks Data ................................ 239

6.5.1 What Are Competing Risks Data? ............... 239

6.5.2 Notation for Competing Risks Data................ 240

6.5.3 Summaries for Competing Risk Data.............. 241

$6.6 \quad$ Some Details ........................................ 247

6.6.1 Bootstrap Confidence Intervals ............... 247

6.6.2 Prediction................................... 248

6.6.3 Adjusting for Nonconfounding Covariates ......... 248

6.6.4 Independent Censoring ......................... 249

$6.6 .5 \quad$ Interval Censoring ............................. 249

6.6.6 Left-Truncation .............................. 250

6.7 Sample Size, Power, and Detectable Effects................. 252

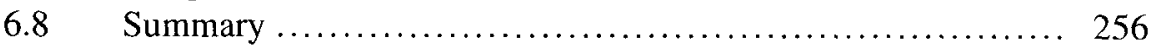

$6.9 \quad$ Further Notes and References ........................ 256

$6.10 \quad$ Problems .......................................... 257

6.11 Learning Objectives................................. 259

7 Repeated Measures and Longitudinal Data Analysis.............. 261

7.1 A Simple Repeated Measures Example: Fecal Fat ............ 262

7.1.1 Model Equations for the Fecal Fat Example ......... 264

7.1.2 Correlations Within Subjects .................... 264

7.1.3 Estimates of the Effects of Pill Type.............. 266

7.2 Hierarchical Data .................................. 267

7.2.1 Example: Treatment of Back Pain ................ 267

7.2.2 Example: Physician Profiling ................. 267

7.2.3 Analysis Strategies for Hierarchical Data ........... 268

$7.3 \quad$ Longitudinal Data ................................ 270

7.3.1 Analysis Strategies for Longitudinal Data .......... 271

7.3.2 Analyzing Change Scores .................... 273

$7.4 \quad$ Generalized Estimating Equations ..................... 276

7.4.1 Example: Birthweight and Birth Order Revisited .... 277

7.4.2 Correlation Structures .......................... 279

7.4.3 Working Correlation and Robust Standard Errors..... 281

7.4.4 Tests and Confidence Intervals .................. 282

7.4.5 Use of xtgee for Clustered Logistic Regression ..... 284

7.5 Random Effects Models ................................... 284

7.6 Re-Analysis of the Georgia Babies Data Set ................ 286 
7.7 Analysis of the SOF BMD Data......................... 288

7.7.1 Time Varying Predictors ........................ 289

7.7.2 Separating Between- and Within-Cluster Information . 291

7.7.3 Prediction.................................. 293

7.7.4 A Logistic Analysis............................ 294

7.8 Marginal Versus Conditional Models ....................... 295

7.9 Example: Cardiac Injury Following Brain Hemorrhage ........ 296

7.9.1 Bootstrap Analysis ............................... 298

7.10 Power and Sample Size for Repeated Measures Designs ........ 301

7.10.1 Between-Cluster Predictor ........................ 301

7.10.2 Within-Cluster Predictor........................ 303

7.11 Summary ............................................ 304

7.12 Further Notes and References .......................... 305

7.12.1 Missing Data ................................. 305

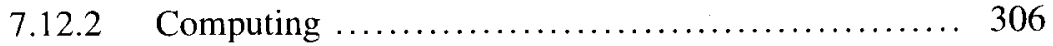

7.13 Problems ............................................ 306

7.14 Learning Objectives................................. 308

$8 \quad$ Generalized Linear Models..................................... 309

8.1 Example: Treatment for Depression ...................... 309

8.1.1 Statistical Issues ............................... 310

8.1.2 Model for the Mean Response .................... 311

8.1.3 Choice of Distribution ........................... 312

8.1.4 Interpreting the Parameters ..................... 312

8.1.5 Further Notes.................................. 313

$8.2 \quad$ Example: Costs of Phototherapy ......................... 314

8.2.1 Model for the Mean Response .................... 315

8.2.2 Choice of Distribution .......................... 315

8.2.3 Interpreting the Parameters ....................... 316

$8.3 \quad$ Generalized Linear Models................................ 316

8.3.1 Example: Risky Drug Use Behavior ............... 317

8.3.2 Modeling Data with Many Zeros .................... 318

8.3.3 Example: A Randomized Trial to Reduce

Risk of Fracture ............................... 321

8.3.4 Relationship of Mean to Variance................. 323

8.3.5 Non-Linear Models ............................. 324

$8.4 \quad$ Sample Size for the Poisson Model ...................... 325

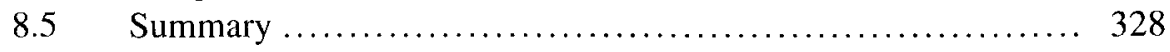

$8.6 \quad$ Further Notes and References ............................ 328

$8.7 \quad$ Problems .............................................. 329

$8.8 \quad$ Learning Objectives................................. 330

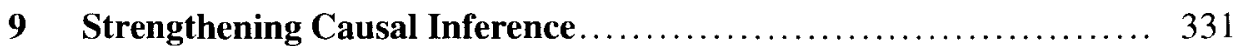

$9.1 \quad$ Potential Outcomes and Causal Effects ....................... 332

9.1.1 Average Causal Effects ......................... 332

9.1.2 Marginal Structural Model ....................... 333 
9.1.3 Fundamental Problem of Causal Inference ........... 333

9.1.4 Randomization Assumption ....................... 334

9.1.5 Conditional Independence ....................... 334

9.1.6 Marginal and Conditional Means ................. 335

9.1.7 Potential Outcomes Estimation .................. 336

9.1.8 Inverse Probability Weighting ................... 337

9.2 Regression as a Basis for Causal Inference .................. 337

9.2.1 No Unmeasured Confounders .................... 338

9.2.2 Correct Model Specification....................... 338

9.2.3 Overlap and the Positivity Assumption............. 338

9.2.4 Lack of Overlap and Model Misspecification ......... 339

9.2.5 Adequate Sample Size and Number of Events ........ 341

9.2.6 Example: Phototherapy for Neonatal Jaundice ......... 341

9.3 Marginal Effects and Potential Outcomes Estimation........... 344

9.3.1 Marginal and Conditional Effects ................. 344

9.3.2 Contrasting Conditional and Marginal Effects ........ 346

9.3.3 When Marginal and Conditional

9.3.4 Potential Outcomes Estimation ..................... 347

9.3.5 Marginal Effects in Longitudinal Data.............. 350

$9.4 \quad$ Propensity Scores ................................... 352

9.4.1 Estimation of Propensity Scores .................... 352

9.4.2 Effect Estimation Using Propensity Scores ........... 355

9.4.3 Inverse Probability Weights ................... 356

9.4.4 Checking for Propensity Score/Exposure Interaction .. 358

9.4.5 Addressing Positivity Violations Using Restriction .... 359

9.4.6 Average Treatment Effect in the Treated (ATT) ....... 360

9.4.7 Recommendations for Using Propensity Scores ...... 362

$9.5 \quad$ Time-Dependent Treatments ............................. 364

9.5.1 Models Using Time-dependent IP Weights .......... 365

9.5.2 Implementation .............................. 367

9.5.3 Drawbacks and Difficulties....................... 368

9.5.4 Focusing on New Users ......................... 369

9.5.5 Nested New-User Cohorts ........................ 370

$9.6 \quad$ Mediation ............................................ 370

9.7 Instrumental Variables ................................. 373

9.7.1 Vulnerabilities............................... 375

9.7.2 Structural Equations and Instrumental Variables...... 377

9.7.3 Checking IV Assumptions ..................... 377

9.7.4 Example: Effect of Hormone Therapy on
Change in LDL.................................. 378

9.7.5 Extension to Binary Exposures and Outcomes ........ 379

9.7.6 Example: Phototherapy for Neonatal Jaundice ........ 380

9.7.7 Interpretation of IV Estimates.................... 382

$9.8 \quad$ Trials with Incomplete Adherence to Treatment ............. 382

9.8.1 Intention-to-Treat .......................... 382 
9.8.2 As-Treated Comparisons by Treatment Received ...... 384

9.8.3 Instrumental Variables ........................ 385

9.8.4 Principal Stratification ....................... 385

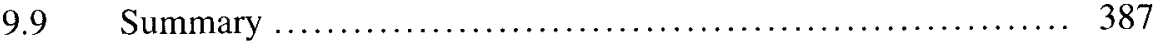

$9.10 \quad$ Further Notes and References ......................... 387

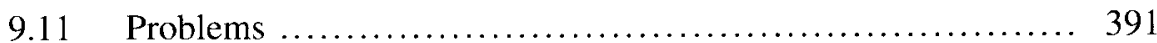

9.12 Learning Objectives................................ 394

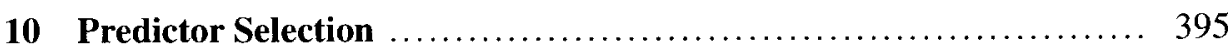

$10.1 \quad$ Prediction .......................................... 396

10.1.1 Bias-Variance Trade-off and Overfitting ........... 397

10.1.2 Measures of Prediction Error...................... 397

10.1.3 Optimism-Corrected Estimates

of Prediction Error ......................... 398

10.1.4 Minimizing Prediction Error Without Overfitting ..... 401

10.1.5 Point Scores ............................... 404

10.1.6 Example: Risk Stratification of Patients

with Heart Disease ................................ 405

10.2 Evaluating a Predictor of Primary Interest.................. 407

10.2.1 Including Predictors for Face Validity .............. 408

10.2.2 Selecting Predictors on Statistical Grounds ........... 408

10.2.3 Interactions With the Predictor of Primary Interest ... 409

10.2.4 Example: Incontinence as a Risk Factor for Falling . . 409

10.2.5 Directed Acyclic Graphs ......................... 410

10.2.6 Randomized Experiments ...................... 416

$10.3 \quad$ Identifying Multiple Important Predictors $\ldots \ldots \ldots \ldots \ldots \ldots \ldots . \ldots 18$

10.3.1 Ruling Out Confounding Is Still Central ........... 418

10.3.2 Cautious Interpretation Is Also Key ............... 419

10.3.3 Example: Risk Factors for Coronary Heart Disease ... 420

10.3.4 Allen-Cady Modified Backward Selection............ 420

10.4 Some Details ............................................ 421

10.4.1 Collinearity ................................. 421

10.4.2 Number of Predictors ........................... 422

10.4.3 Alternatives to Backward Selection ................. 424

10.4.4 Model Selection and Checking.................. 425

10.4.5 Model Selection Complicates Inference ............. 425

10.5 Summary ...................................... 427

10.6 Further Notes and References .......................... 427

10.7 Problems ............................................... 428

$10.8 \quad$ Learning Objectives................................. 429

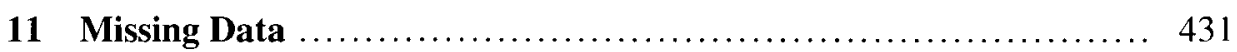

11.1 Why Missing Data Can Be a Problem ................... 432

11.1.1 Missing Predictor in Linear Regression ............ 432

11.1.2 Missing Outcome in Longitudinal Data ............ 434 
11.2 Classifications of Missing Data ........................ 437

11.2.1 Mechanisms for Missing Data .................... 438

11.3 Simple Approaches to Handling Missing Data ............... 442

11.3.1 Include a Missing Data Category .................. 442

11.3.2 Last Observation or Baseline Carried Forward ........ 442

11.4 Methods for Handling Missing Data......................... 444

11.5 Missing Data in the Predictors and Multiple Imputation......... 444

11.5.1 Remarks About Using Multiple Imputation .......... 446

11.5.2 Approaches to Multiple Imputation .............. 447

11.5.3 Multiple Imputation for HERS ................... 449

11.6 Deciding Which Missing Data Mechanism May Be Applicable ................................... 451

11.7 Missing Outcomes, Missing Completely at Random .......... 452

11.8 Missing Outcomes, Covariate-Dependent Missing Completely at Random ................................. 452

11.9 Missing Outcomes for Longitudinal Studies, Missing at Random ................................ 453

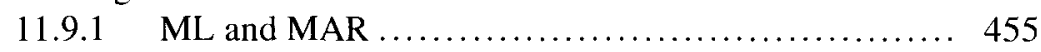

11.9.2 Multiple Imputation ....................... 456

11.9.3 Inverse Probability Weighting ................... 456

11.10 Technical Details About Maximum Likelihood and Data Which are Missing at Random...................... 458

11.10.1 An Example of the EM Algorithm ................ 458

11.10.2 The EM Algorithm Imputes the Missing Data ....... 460

11.10.3 ML Versus MI with Missing Outcomes ............. 461

11.11 Methods for Data that are Missing Not at Random ........... 461

11.11.1 Pattern Mixture Models ........................... 461

11.11.2 Multiple Imputation Under MNAR ............... 463

11.11.3 Joint Modeling of Outcomes and the
Dropout Process $\ldots \ldots \ldots \ldots \ldots \ldots \ldots \ldots \ldots \ldots \ldots \ldots, 463$

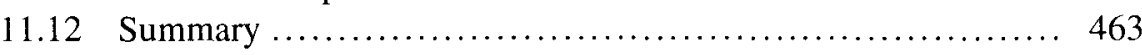

11.13 Further Notes and References .......................... 464

11.14 Problems .............................................. 465

11.15 Learning Objectives................................ 467

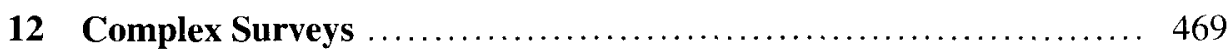

12.1 Overview of Complex Survey Designs .................... 470

12.2 Inverse Probability Weighting ...................... 471

12.2.1 Accounting for Inverse Probability Weights

in the Analysis ........................... 473

12.2.2 Inverse Probability Weights and Missing Data ....... 473

$12.3 \quad$ Clustering and Stratification ............................ 474

12.3.1 Design Effects.......................... 474

12.4 Example: Diabetes in NHANES $\ldots \ldots \ldots \ldots \ldots \ldots \ldots \ldots \ldots \ldots, 475$ 
$12.5 \quad$ Some Details ........................................ 477

12.5.1 Ignoring Secondary Levels of Clustering ............ 477

12.5.2 Other Methods of Variance Estimation .............. 477

12.5.3 Model Checking .............................. 478

12.5.4 Postestimation Capabilities in Stata................ 478

12.5.5 Other Statistical Packages for Complex Surveys ....... 479

12.6 Summary ............................................ 479

12.7 Further Notes and References ............................. 479

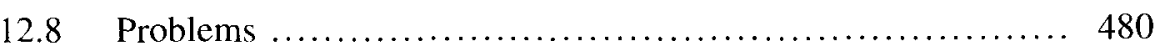

$12.9 \quad$ Learning Objectives................................. 480

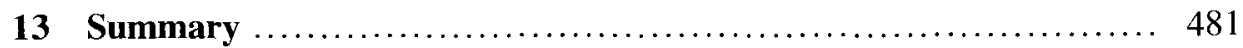

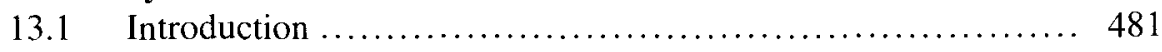

13.2 Selecting Appropriate Statistical Methods.................. 482

13.3 Planning and Executing a Data Analysis ................... 483

13.3.1 Analysis Plans ............................... 483

13.3.2 Choice of Software ........................... 484

13.3.3 Data Preparation ............................... 484

13.3.4 Record Keeping and Reproducibility of Results ...... 484

13.3.5 Data Security ................................... 485

13.3.6 Consulting a Statistician ......................... 485

13.3.7 Use of Internet Resources ...................... 486

13.4 Further Notes and References .......................... 486

13.4.1 Multiple Hypothesis Tests ...................... 486

13.4.2 Statistical Learning ........................... 487

References $\ldots \ldots \ldots \ldots \ldots \ldots \ldots \ldots \ldots \ldots \ldots \ldots \ldots \ldots \ldots \ldots \ldots \ldots \ldots \ldots \ldots, 489$

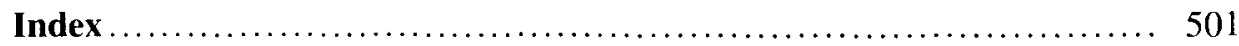

